\title{
Performance comparison of wearable-based pedestrian navigation systems in large areas
}

\author{
Dina Bousdar Ahmed* and Luis Enrique Díez Blanco ${ }^{\dagger}$ and Estefania Munoz Diaz* \\ ${ }^{*}$ German Aerospace Center (DLR) \\ Institute of Communications and Navigation, Munich, Germany \\ Email: \{Dina.BousdarAhmed, Estefania.Munoz\}@dlr.de \\ $\dagger$ Faculty of Engineering, University of Deusto \\ Bilbao, Spain \\ Email: luis.enrique.diez@deusto.es
}

\begin{abstract}
Wearable devices are a key driver for the development of pedestrian navigation systems. In this work, we consider inertial navigation systems (INSs). There is a diversity of such INSs. Normally, the comparison of INSs is restricted to indoor environments, or to outdoor small areas. However, it is of interest to study the behaviour of INSs in large areas. To that end, we present a ground truth system with $\mathrm{cm}$ accuracy to evaluate navigation systems. The ground truth system is distributed in an area of $14380 \mathrm{~m}^{2}$ approximately. The ground truth system is used to evaluate three INSs based on three different body locations: the thigh, which is denoted as pocket, the wrist and the foot. Additionally, the data from a glasses-mounted inertial measurement unit (IMU) are also collected. The data, as well as the ground truth, have been made available for download. The results of evaluating 995 ground truth points indicate that the foot INS outperforms the pocket INS in, at most, $2 \mathrm{~cm} / \mathrm{s}$. The pocket INS has, in contrast, a better standard deviation of the position error, and a robust step detection. The wrist INS is the most sensitive system to outliers. Therefore, its average position error is the highest. All in all, there is still room for improvement in the performance of all evaluated INSs.
\end{abstract}

\section{INTRODUCTION}

Wearable devices are a key driver for the development of pedestrian navigation systems. These devices have the necessary sensory and processing capabilities [1] to implement such systems. Furthermore, wearable devices are worn comfortably and they can be integrated in the clothes. According to Vandrico's Wearable Database [2], wrist-worn wearable devices are the most numerous in the market. However, new devices are released continually for other body locations, e.g. torso, legs, feet, head.

Each body location is an opportunity to develop a new navigation system. In our case, we consider systems based on inertial sensors. The sensor's body location ranges from foot-mounted [3], to pocket-mounted [4], chest-mounted [5], wrist-mounted [6], head-mounted [7], etc. INSs differ not only in the body location of the sensor, but also in the pedestrian dead reckoning (PDR) algorithm.

The diversity of INSs results in the fact that each one has certain advantages over the others. The identification of the latter is only possible if the systems are tested under the same conditions, similarly to [8]. In previous work, we have compared a foot-mounted INS and a pocket-mounted INS [9].

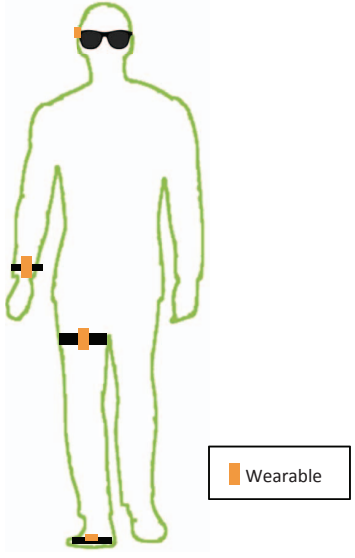

Fig. 1: IMUs locations used in the walks.

In the state of the art, the comparison of INSs is either restricted to indoor environments, [8], or to outdoor small areas [9]. The evaluation of INSs in large areas is, to the best of our knowledge, not performed in the state of the art. The reason is that INSs suffer from cumulative errors that disturb rapidly the estimated odometry of the pedestrian. Therefore, large areas are not tested with INSs because the results are often unsatisfactory. However, we consider of interest to study the behaviour of INSs in such scenarios. The study will allow to assess, for example, if one INS behaves better in the long term than another one.

The ground truth system is also relevant for a fair comparison. There are two main alternatives used in the state of the art. The first one is the qualitative comparison of the odometry to the map where the walk is performed. The second one is to start and end the walk at the same point. The comparison is to compute the Euclidean distance between the start and end point estimated by the odometry. Although useful, neither of latter two approaches provides a meaningful quantitative evaluation of the performance of an INS.

Furthermore, it is frequently the case that the data sets used in the experiments are not available for public use. Therefore, besides the final results and conclusions, there is little benefit for the scientific community from the experimental work done 
by other peers.

We contribute to the state-of-the-art with:

- The qualitative and quantitative evaluation of three existing INSs in a large area, approximately $14380 \mathrm{~m}^{2}$.

- The identification of the strengths and weaknesses of the INSs under evaluation.

- The publication of the data set, as well as the ground truth, used in the experiments [10].

\section{InERTial NAVIGATION SyStemS}

The INSs evaluated in this work are based on a pocketmounted IMU, wrist-mounted IMU and foot-mounted IMU, see Fig. 1. In addition, the inertial measurements of an IMU mounted on the glasses have been collected. The detailed description of the INSs under test is out of the scope of this paper. However, in the following, an overview of each one is presented for completeness of this work.

\section{A. Pocket INS}

The upper thigh is, for several reasons, an attractive position to implement INSs. Firstly, phases of the gait cycle can be clearly observed by tracking the motion of the leg. Secondly, walking in flat surfaces and walking up/down stairs can be detected by solely analyzing the thigh's inertial measurements, [4]. Finally, devices such as smartphones are often carried in the trousers' pocket. Thus, they can be used to implement such INSs.

The pocket INS used in this work was first presented in [11] and [12] offers a more detailed analysis. The pocket INS uses an IMU mounted on the user's upper thigh. Furthermore, the step-length-and-heading estimation approach is used to estimate the user's position. The step detector and step length estimator are presented in [4], [13]. The pitch angle of the upper thigh is used to detect steps by identifying its maximum peaks and the amplitude of the pitch angle estimation is related to the step length. For further details, the reader is referred to the aforementioned papers and references therein. It is worth clarifying that the steps detected by the pocket INS are, in the context of the gait cycle, the strides of the leg where the IMU is mounted.

\section{B. Wrist INS}

The wrist is a body location with great potential for the implementation of INSs. For instance, it is a convenient sensor location because many users wear watches or bands on a daily basis. There are already smart watches and smart bands [2] that enable the implementation of INSs. Furthermore, it is possible to detected multiple daily activities from the wrist. These activities can be used to add context to the navigation and to develop new location-based services.

The wrist-worn INS tested in this work is based on the proposal of Qian et al. [14]. Modifications, which are described in [6] and references therein, were made to that proposal. The wrist INS is composed of the classic modules of a step-and-heading based pedestrian dead reckoning system. In addition, the wrist INS tested here was presented to the Indoor

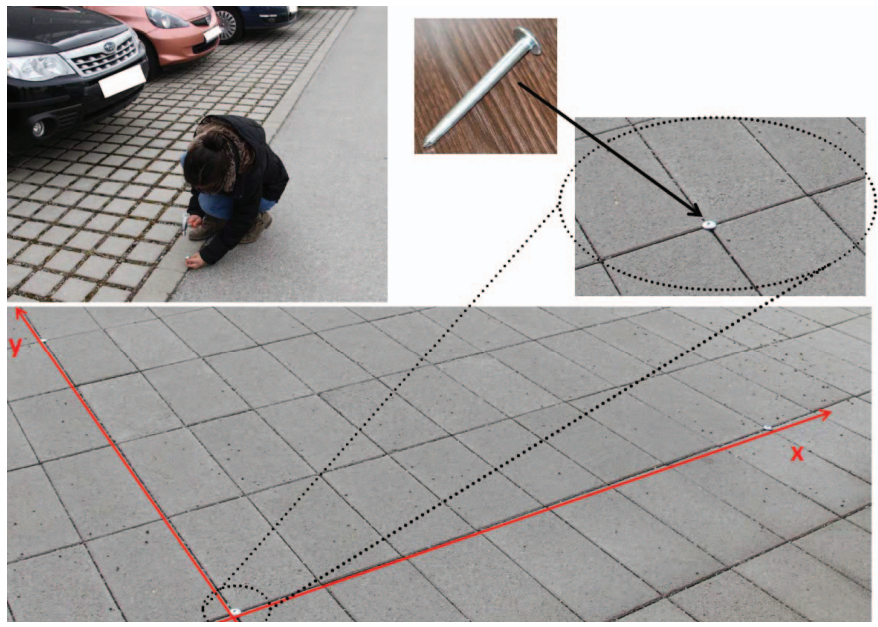

Fig. 2: Origin of coordinates and hammering of the nails in the ground.

Localization Competition of the Indoor Positioning and Indoor Navigation (IPIN) conference. The latter took place in October 2016 in Alcalá de Henares, Spain. The wrist INS detects, in the context of the gait cycle, the steps, i.e. whenever either left or right foot hits the floor.

\section{Foot INS}

The foot is a very convenient body location to implement INS systems. The strapdown algorithm with inertial measurements can only be used if the IMU is mounted on the foot. The reason is that the observation of the foot's stance phase allows for zero-velocity updates, which reduces significantly the cumulative error in PDR algorithms.

The foot INS used in this work is described in [15] and references therein. The foot INS processes the inertial measurements of the foot with a strapdown algorithm that is implemented through an unscented Kalman filter. In addition, the stance phase detection is based on the proposal of Ruppelt et al. [16]. Regarding the gait cycle, the foot INS detects stance phases of the foot where the IMU is mounted, i.e. one stance phase per gait cycle.

\section{EXPERIMENT SET UP}

\section{A. Ground truth system}

The ground truth system comprises a set of points, whose location is known accurately. These points will be referred to as ground truth points (GTPs), and they are visited during the experiments. The position estimated by the INSs for the GTPs, which will be named marker, is compared to its true position. The comparison allows, therefore, to evaluate the performance of the INSs.

The GTPs are indicated by nails that were hammered in the ground for the purpose of this work, see Fig. 2. Once the GTPs are indicated by the nails, the next step is to measure their position. For this purpose, the Leica tachymeter was used to create a local frame. Fig. 3 shows the Leica tachymeter and the prism located on top of a GTP. The origin and orientation 

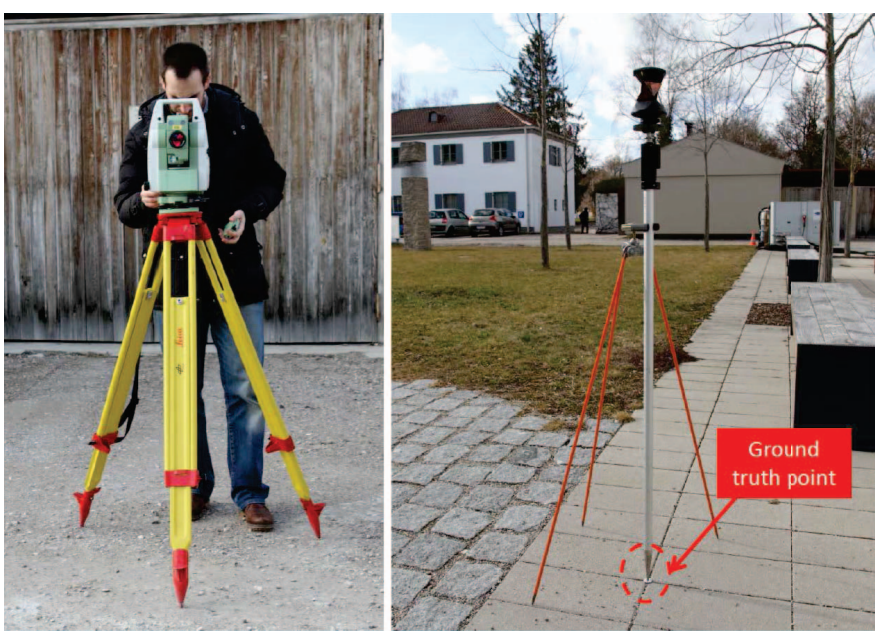

Fig. 3: (Left) Leica tachymeter. (Right) $360^{\circ}$ prism located on top of a GTP.

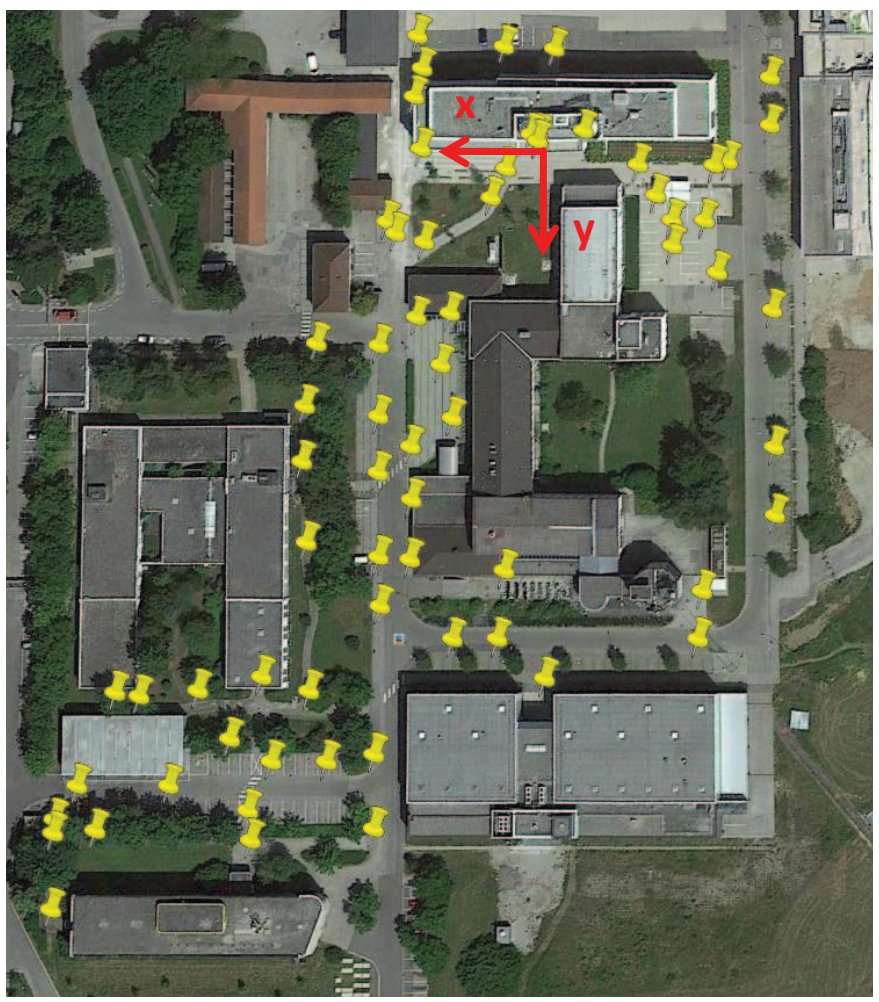

Fig. 4: GTPs distributed in the test area of $14380 \mathrm{~m}^{2}$ approximately. The map of the area was taken from Google Earth.

of the local frame must be indicated to the tachymeter. In this case, we have set the origin and direction of the $x-y$ axis as indicated in Fig. 4.

In the end, the ground truth system created in this work comprises 69 GTPs whose location is known with $\mathrm{cm}$ accuracy distributed in an area of $14380 \mathrm{~m}^{2}$, see Fig. 4. In the latter, the GTPs are indicated by yellow pins.

In order to use the ground truth system, a program was implemented to run on a Raspberry Pi. The program logs

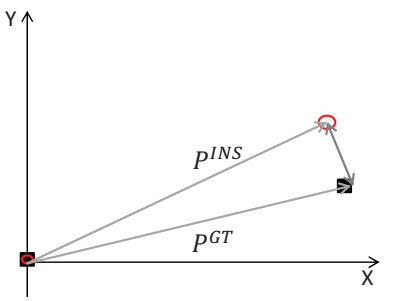

- GTP

- Position estimated by the INS

Fig. 5: Position error between the true GTP position $\left(P^{G T}\right)$ and the estimated GTP position $\left(P^{I N S}\right)$.

both the data from multiple IMUs, including an absolute time stamp, and stores an absolute time stamp when a GTP is crossed. In order to signal that a GPT is crossed, a board with multiple buttons and LED indicators is developed and attached to the Raspberry Pi, see Fig. 6. There are several advantages about using the Raspberry Pi in Fig. 6. The Raspberry is easy to use, it is portable and the design modifications allow a practical recording of the visited GTPs.

The estimated position of each marker is compared to its true position, see Fig. 5. The error in position is normalized by the elapsed time. Therefore, the error metric $\left(e_{P}\right)$ is defined, for the time instant $k$, as follows:

$$
e_{P}(k)=\frac{\left|P^{G T}(k)-P^{I N S}(k)\right|}{t_{k}},
$$

where $P^{G T}(k)$ denotes the true position vector of the GTP visited at the $k$-th time, $P^{I N S}(k)$ denotes the estimated position vector of the GTP visited at the $k$-th time, $|\cdot|$ denotes the norm of a vector and $t_{k}$ denotes the elapsed time at the $k$-th time.

\section{B. Sensor set up}

The sensors set up is shown in Fig. 6. A total of 4 IMUs are placed on the glasses, the wrist, the upper thigh and the foot respectively. The IMUs are connected by a cable to a Raspberry Pi. Although only the inertial measurements from the pocket-mounted, wrist-mounted and foot-mounted IMU are used in this work, the sensor on the glasses is also included to collect more data and make it available to the public.

Regarding the sensors, four MTw new generation manufactured by Xsens have been used. These devices include a 3D-accelerometer, a 3D-gyroscope, a 3D-magnetometer and they can also measure pressure and the sensor's temperature. The Allan variance analysis [17] has been run on the accelerometers and gyroscopes to guarantee that all IMUs have the same quality. This analysis is necessary to guarantee a fair comparison between INSs. The random walk and bias stability of each axis of each accelerometer and gyroscope have been estimated. The results showed that all sensors have the same quality. Nevertheless, the tables with the values of the randon walk and bias stability are skipped for the sake of simplicity.

\section{Walks}

The data from the IMUs have been recorded at a frequency of $100 \mathrm{~Hz}$ for each walk. The following information is recorded 


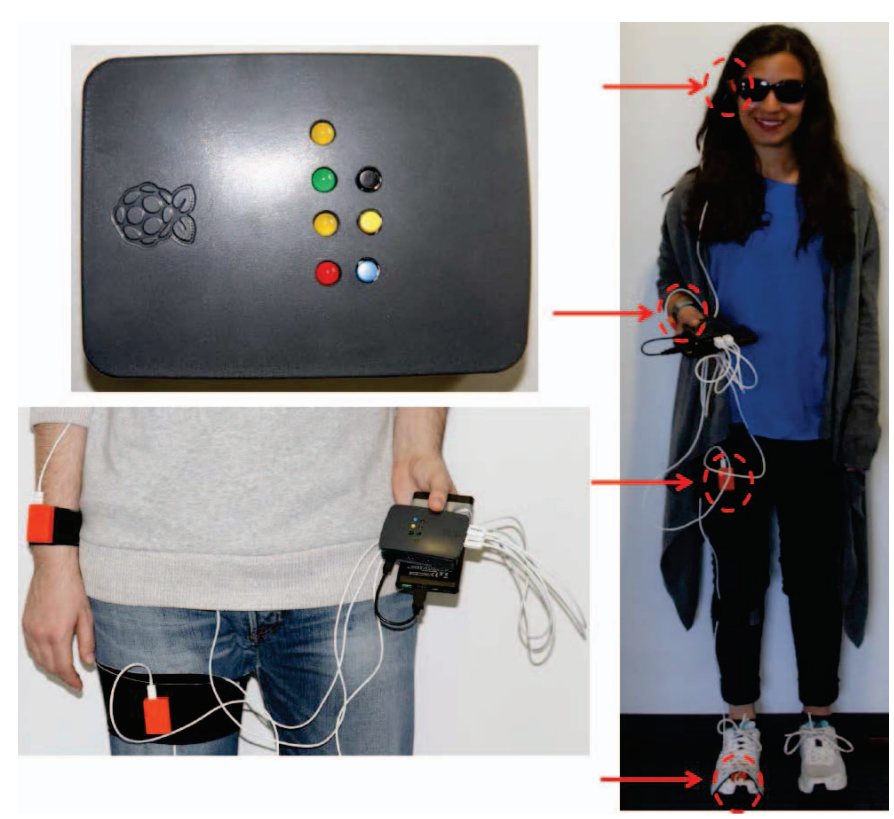

Fig. 6: (Top left) Raspberry Pi module used to record the data from the IMUs. (Bottom left) From left to right, sensor on wrist and thigh and Raspberry Pi used to log the data and time stamps when the GTPs are crossed. (Right) Sensor set up on glasses, wrist, thigh and foot.

TABLE I: Summary table of the experiments.

\begin{tabular}{ccc}
\hline Total no. of walks & Total time & Total distance \\
\hline 29 & $4 h 51 \mathrm{~min}$ & $20 \mathrm{~km}$ \\
\hline
\end{tabular}

for each sensor in each walk: an absolute time stamp, a sequence number, 3D-acceleration, 3D-turn rate, 3D-magnetic field. Additionally, a list of time stamps, that indicate when the user passed a GTP, is also stored for each walk.

Regarding the walks, the GTPs to visit in each walk were decided prior to performing the walk. The users would signal, by pressing the blue button of the Raspberry Pi on Fig. 6, each time they walked on top of a GTP. The users were indicated, prior to performing the walks, not stop on top of the GTPs when pressing the button. The reason was to favor natural walks. During the experiments, the wrist IMU was mainly swinging. Nevertheless, there were some outliers. Additionally, when indoor and outdoor areas were combined in a single walk, the users opened the doors with the hand where the wrist IMU was located. The users were allowed to move the head freely during the walks.

Table I indicates the total number of walks, as well as the approximate total time and distance walked. The length of the walks ranges from $0.2 \mathrm{~km}$ to $1.9 \mathrm{~km}$ approximately.

\section{EVALUATION}

\section{A. Statistics}

Table II presents the error statistics of the pocket INS, the wrist INS and the foot INS. In addition, the number of detected
TABLE II: Mean $(\mu)$ and standard deviation $(\sigma)$, written as $\mu \pm \sigma$, of the position error $e_{P}$ of 29 walks with a total of 995 GTPs. The number of steps detected is also shown.

\begin{tabular}{cccc}
\hline & Pocket INS & Wrist INS & Foot INS \\
\hline $\boldsymbol{e}_{\boldsymbol{P}}[\boldsymbol{m} / \boldsymbol{s}]$ & $0.19 \pm 0.15$ & $0.30 \pm 0.70$ & $0.19 \pm 0.21$ \\
Steps/strides detected & 13880 & 23195 & 14872 \\
\hline
\end{tabular}

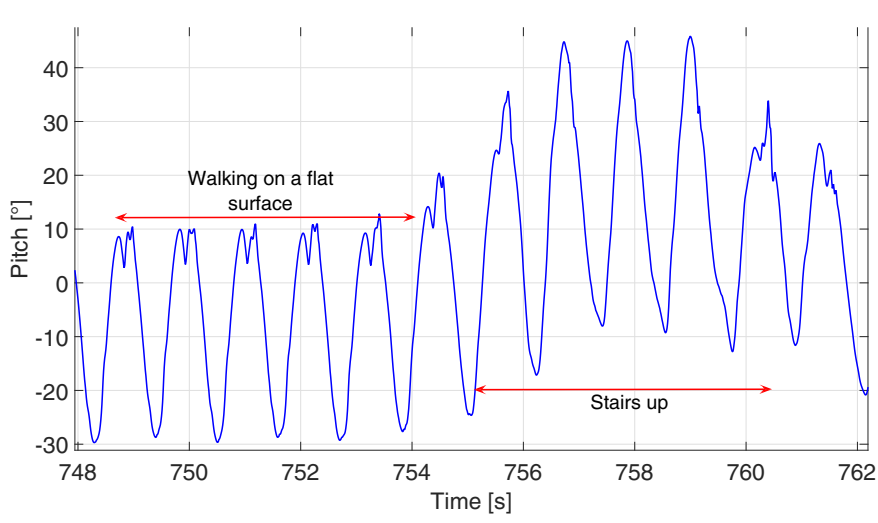

Fig. 7: Pitch angle of the leg during a walk when the user had to walk 5 stairs up.

steps of each INS is also indicated. The following sections discuss the results presented in Table II.

\section{B. Discussion}

1) PDR algorithm: as described in section II, the pocket INS and wrist INS are based on a step-length-and-heading estimation approach. A requirement of the latter is the use of a model to estimate the step length. Therefore, the quality of the step length estimate depends on the better fit of the model to estimate the step length. In contrast, the foot INS is based on the strapdown algorithm. The length estimations are done by double integration of the foot's acceleration. Thus, the quality of the length estimate is affected by the cumulative errors.

Although the evaluation of the 3D performance of the INSs is out of the scope of this paper, it is worth highlighting that the strapdown algorithm can estimate 3D position. In contrast, PDR based in the step-length-and-heading estimation approach is limited to 2D scenarios when only the IMU data is used. Nevertheless, stairs can be clearly observed by tracking the pitch angle of the leg. Fig. 7 presents the pitch angle of the leg in a case when the user had to walk 6 stairs up. Fig. 7 shows how the values of the pitch angle are different when the user is walking on a flat surface than when walking stairs. This property can be used to track 3D-position with the pocket INS [4].

2) Step detection: according to Section II, the number of steps detected by the pocket INS should be the same as the number of stance phases detected by the foot INS. In addition, both of the previous parameters should be half of the number of steps detected by the wrist INS. Table II shows that the pocket INS detected a total of 13880 steps. In [4], the pocket INS is proven to have detected correctly all steps, which is 


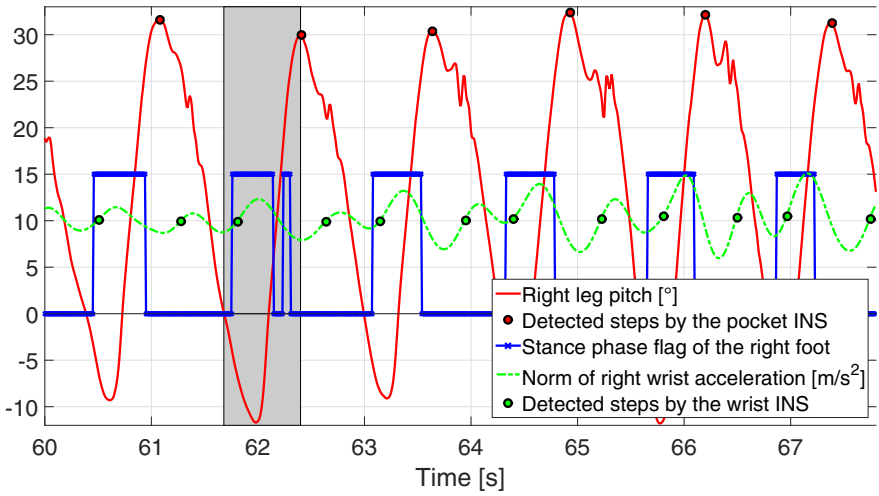

Fig. 8: Comparison of the signals that each INS uses to either detect strides (pocket INS), steps (wrist INS), or detect stance phases (foot INS). The shadowed area indicates a period of time when the foot INS detects two stance phased instead of one.

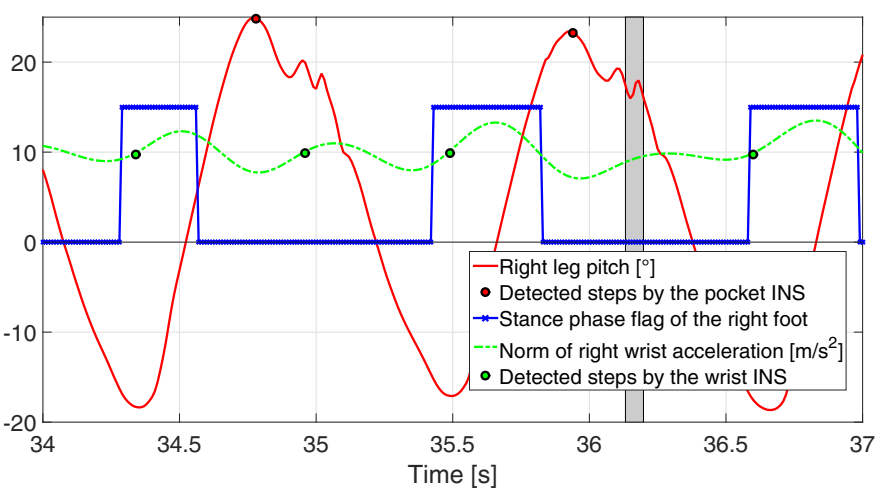

Fig. 9: Comparison of the signals that each INS uses to either detect strides (pocket INS), steps (wrist INS), or detect stance phases (foot INS). The shadowed area indicates an undetected step by the wrist INS.

again proven in [9]. Therefore, 13880 is taken as the true number of steps. Thus, the number of steps detected by the wrist INS and the foot INS are compared to the pocket INS's detected steps, i.e. 13880.

According to Table II, the foot INS detects 992 steps more than the pocket INS. The difference is because the step detection algorithm of the foot INS fails to detect some intervals of the foot's stance phase, see Fig. 8. These misdetections result in the foot INS accounting more stance phases than the true number of them. The mis-detections affect the foot INS during the measurement update stage of the Kalman filter that this INS implements. In fact, failure to detect stance phases causes the filter not to apply velocity corrections, when indeed, the update could be done [9].

Table II indicates that the wrist INS detects 23195 steps, i.e. approximately 11597 steps as defined by the pocket INS. Therefore, the wrist INS detects 3275 steps less than the pocket INS. These mis-detections are caused by undetected steps, false negatives, as the shadowed area in Fig. 9 shows.

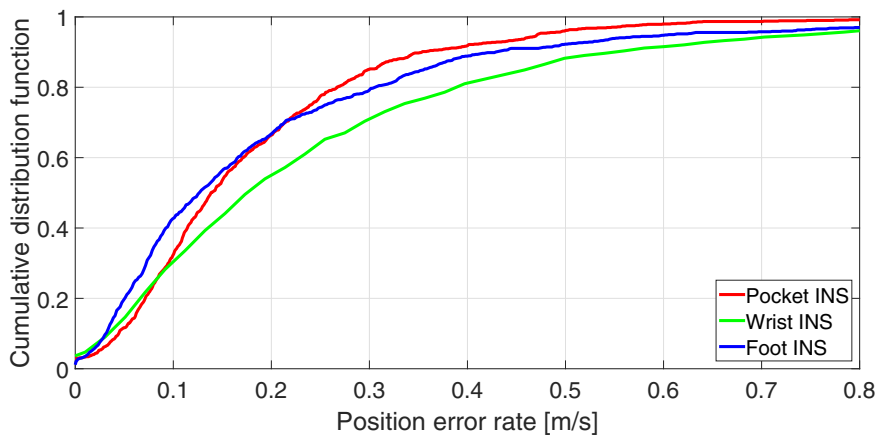

Fig. 10: Cumulative distribution function of the position error of each INS.

However, outliers during the walk can trigger the false detection of steps, e.g. opening a door.

3) Distance and orientation error: the position error rate in equation (1) evaluates the performance of the INSs regarding the accuracy of the position estimate. This accuracy depends simultaneously on the accuracy of the length estimate and orientation estimate of the INS.

The results in Table II indicate that the pocket INS and foot INS have the same average error. However, the pocket INS has a lower standard deviation. Generally, the length estimate of the foot INS is better than the pocket INS's and the wrist INS's. The reason is that the foot INS does not rely on a model for the length estimate. Therefore, the better performance of the pocket INS relies on its orientation estimate.

Regarding the wrist INS, the results show that it has the highest average error and standard deviation. This is an expected result because the wrist INS is the most sensitive system to both the way the user walks and to outliers. On the one hand, the way each user swings the arm while walking might benefit or affect the performance of the wrist INS. On the other hand, outliers like opening doors, scratching, fixing one's hair, etc. result in a degradation of the INS performance. Although these outliers affect the performance of the INS, they provide useful contextual information. In fact, they can only be observed thanks to the wrist IMU.

Fig. 10 presents the cumulative distribution function (CDF) of the position error rate in equation (1). The CDF indicates the probability that the position error rate $\left(e_{P}\right)$ takes values less than or equal to a certain value. For example, let us consider the $80 \%$ value of the CDF. In this case, the error of the pocket INS is, with a probability of $80 \%$, under $28 \mathrm{~cm} / \mathrm{s}$ approximately. The error of the foot INS is, with an $80 \%$ probability, under $30 \mathrm{~cm} / \mathrm{s}$, whereas the error of the wrist INS is under $42 \mathrm{~cm} / \mathrm{s}$ for the aforementioned probability.

It can be seen, from Fig. 10, that the foot INS outperforms the pocket INS in $2 \mathrm{~cm} / \mathrm{s}$ up to $40 \%$ of the cases. In fact, the foot INS outperforms the pocket INS until an error of $25 \mathrm{~cm} / \mathrm{s}$. The latter happens with a probability of $75 \%$. That is, the foot INS outperforms the pocket INS in, as much, $75 \%$ of the cases. In the remaining ones, the pocket INS has better position error rate than the foot INS. 


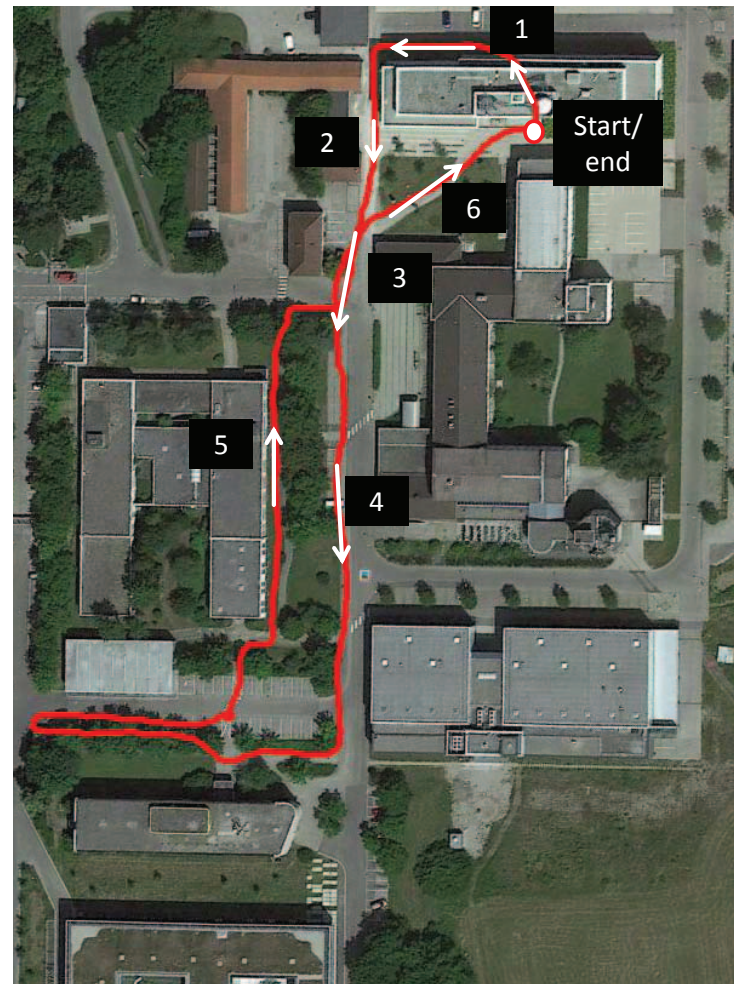

Fig. 11: Approximate walked path. The start and end points are indicated by the circle marker. The sequence of the walk is indicated by the white arrows. The map of the site was taken from Google Earth.

Fig. 11 presents the approximate path of one walk. The path is approximate because the users were indicated only which GTPs to visit, and not the exact path to follow. The odometry estimated by each INS is presented in Fig. 12. The position error rate metric (1) of this walk is presented in Fig. 13. It can be seen, from Fig. 13, that the pocket INS and foot INS have a small position error in comparison to the wrist INS. This is true until $70 s$ approximately. After $70 s$, the error increases faster for the pocket INS and foot INS than for the wrist INS. This behaviour holds until approximately $200 s$. From that time on, the position error rate decreases. The decrease is caused by the shape of the path, see Fig. 11. The position error rate in the way back, i.e. from $250 \mathrm{~s}$ on, is smaller than the maximum position error rate. The latter occurs at different times for each INS. For instance, the maximum position error rate of the pocket INS occurs around markers 67-68.

All in all, the results show that the average position error rate is $19 \mathrm{~cm} / \mathrm{s}$ within a set of walks that are up to $1.9 \mathrm{~km}$, see Table II. Therefore, there is yet room for improvement in the performance of the evaluated INSs. Improvement measures should address three main issues. The first one is the drift in the orientation estimation. The second one is the accuracy of the model for step length estimation in approaches that use such a model. The third one is the correct detection of stance phases and/or steps. Table III summarizes the main advantages and disadvantages of each INSs evaluated in this work.

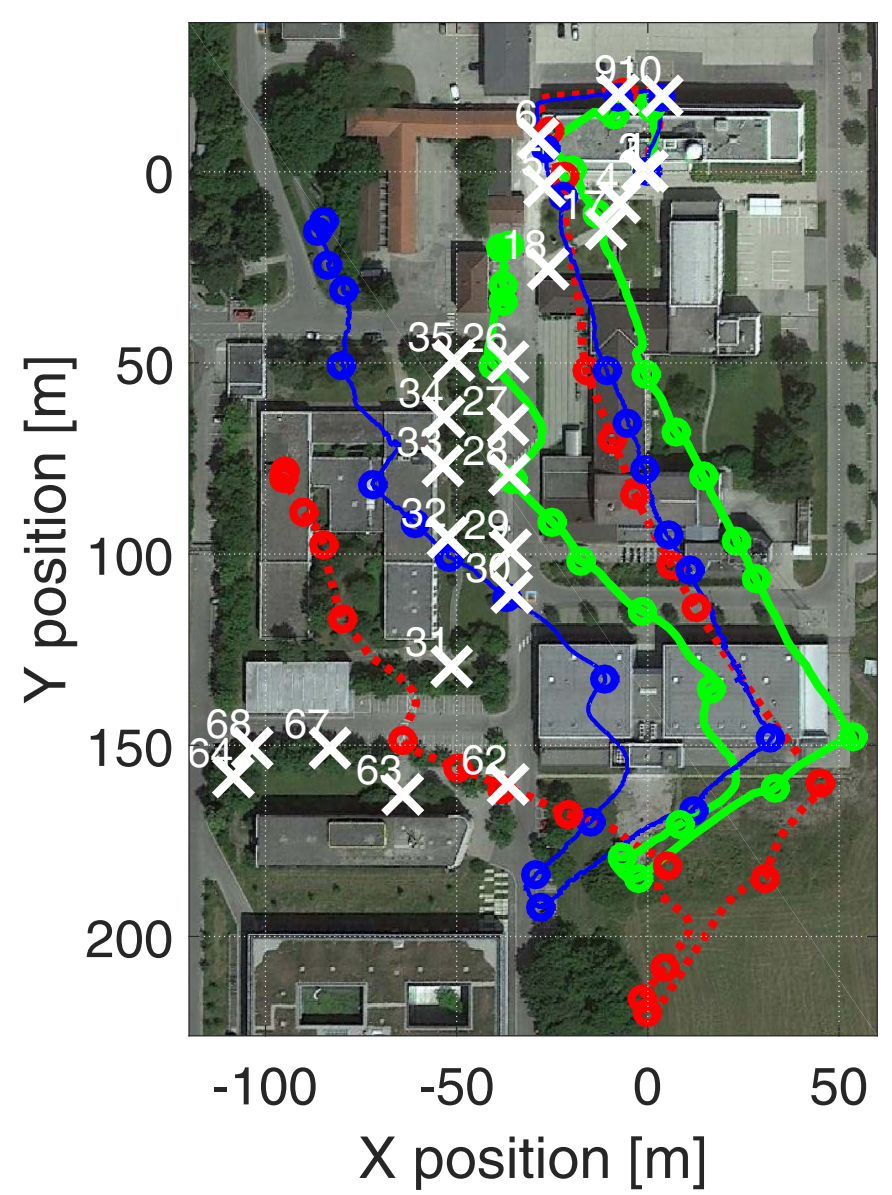

Fig. 12: Odometry estimated by the pocket INS (red-dotted line), the wrist INS (green-thick-solid line) and the foot INS (blue-thin-solid line). The $\mathrm{x}$-marks indicate the true position of the GTPs. The circle marks indicate the position estimated by the INSs for the GTPs. The map of the site was taken from Google Earth.

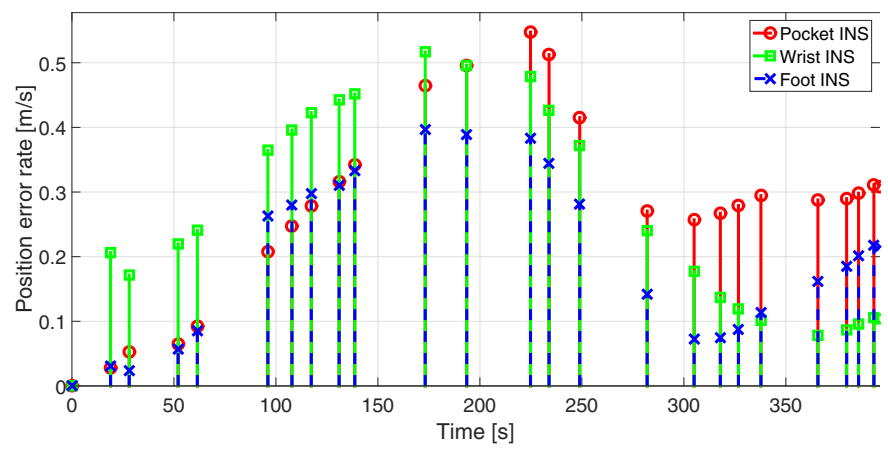

Fig. 13: Position error rate of the walk in Fig. 12. 
TABLE III: Summary table of the identified advantages and disadvantages of the INSs under evaluation.

\begin{tabular}{lll}
\hline & \multicolumn{1}{c}{ Advantages } & \multicolumn{1}{c}{ Disadvantages } \\
\hline \multirow{2}{*}{ Pocket INS } & $\begin{array}{l}\text { Stairs detection (2.5D). } \\
\text { Robust step detection. } \\
\text { Good orientation estimate. }\end{array}$ & $\begin{array}{l}\text { Model-based estimation } \\
\text { of step length. }\end{array}$ \\
\hline \multirow{2}{*}{ Wrist INS } & $\begin{array}{l}\text { Contextual information } \\
\text { about the users activity, } \\
\text { e.g. opening doors. }\end{array}$ & $\begin{array}{l}\text { Model-based step length } \\
\text { estimation. } \\
\text { Outliers affect the accu- } \\
\text { racy of the position. }\end{array}$ \\
\hline \multirow{2}{*}{ Foot INS } & $\begin{array}{l}\text { 3D-positioning. } \\
\text { Non-dependency on a } \\
\text { model for step length } \\
\text { estimation. }\end{array}$ & $\begin{array}{l}\text { Missed ZUPTs. } \\
\text { Accumulated errors of the } \\
\text { inertial measurements af- } \\
\text { fect the position accuracy. }\end{array}$ \\
\hline
\end{tabular}

\section{CONCLusion}

In this work, we have presented a ground truth system with $\mathrm{cm}$ accuracy to evaluate navigation systems. The ground truth system is distributed in a large area, which constitutes a challenging scenario for inertial navigation systems. Three INSs based on a pocket-mounted IMU, a wrist-mounted IMU and a foot-mounted IMU have been evaluated with the proposed ground truth system.

The results show that the pocket INS is able to detect the stairs that were encountered during the walk. Furthermore, the pocket INS outperforms the foot INS in the standard deviation of the error in position. Nevertheless, the foot INS outperforms the pocket INS up to $75 \%$ of the cases. The results show that the wrist INS is the most sensitive system to outliers. The latter are caused by other motions of the hand than swinging, e.g. opening a door. On the good side, the wrist outliers provide contextual information about the user's activity. This information could be used to extend the functionality of the wrist INS.

\section{REFERENCES}

[1] What is a wearable device? [Online]. Available: http://www.wearabledevices.com/what-is-a-wearable-device/

[2] Vandrico Inc., "Wearable technology database," Online, 2017. [Online]. Available: http://vandrico.com/wearables/

[3] A. R. Jimnez, F. Seco, J. C. Prieto, and J. Guevara, "Indoor pedestrian navigation using an INS/EKF framework for yaw drift reduction and a foot-mounted IMU," in 2010 7th Workshop on Positioning, Navigation and Communication, March 2010, pp. 135-143.

[4] E. Munoz Diaz, "Inertial pocket navigation system: Unaided 3D positioning," Sensors (Switzerland), vol. 15, no. 4, pp. 9156-9178, 2015.

[5] T. N. Do, R. Liu, C. Yuen, M. Zhang, and U. X. Tan, "Personal dead reckoning using IMU mounted on upper torso and inverted pendulum model,' IEEE Sensors Journal, vol. 16, no. 21, pp. 7600-7608, Nov 2016.

[6] L. E. Díez, A. Bahillo, S. Bataineh, A. D. Masegosa, and A. Perallos, "Enhancing improved heuristic drift elimination for step-and-heading based pedestrian dead-reckoning systems," in 38th Annual International Conference of the IEEE Engineering in Medicine and Biology Society (EMBC), Aug 2016, pp. 4415-4418.

[7] J. Windau and L. Itti, "Walking compass with head-mounted IMU sensor," in 2016 IEEE International Conference on Robotics and Automation (ICRA), May 2016, pp. 5542-5547.

[8] D. Finker, J. Kocjan, J. Rutkowski, and R. Cai, "Evaluation of an autonomous navigation and positioning system for IAEA safeguards inspectors," pp. 111-119, 2014.
[9] D. Bousdar, E. Munoz Diaz, and S. Kaiser, "Performance comparison of foot- and pocket-mounted inertial navigation systems," in International Conference on Indoor Positioning and Indoor Navigation, October 2016.

[10] "Data set of walks in a large area," May 2017, user name for access: PDR_Walks_LargeAreas_guest, password: $x U 4 n V w u z$, port numbers: 20-21. (Only for reviewers). [Online]. Available: ftp.dlr.de

[11] E. Munoz Diaz, A. L. Mendiguchia Gonzalez, and F. de Ponte Müller, "Standalone inertial pocket navigation system," in Proceedings of the IEEE/ION Position Location and Navigation Symposium, Monterey, USA, May 2014.

[12] E. Munoz Diaz, A. Jimenez, F. de Ponte Müller, and F. Zampella, "Evaluation of AHRS algorithms for inertial personal localization in industrial environments," in Proceedings of the IEEE International Conference on Industrial Technology (ICIT), Seville, Spain, March 2015.

[13] E. Munoz Diaz and A. L. Mendiguchia Gonzalez, "Step detector and step length estimator for an inertial pocket navigation system," in Proceedings of theIEEE/ION Position Location and Navigation Symposium (PLANS), South Korea, October 2014.

[14] J. Qian, J. Ma, L. Xu, R. Ying, W. Yu, , and P. Liu, "Investigating the use of MEMS based wrist-worn IMU for pedestrian navigation application," in 26th International Technical Meeting, ION GNSS 2013, Institute of Navigation, Ed., 2013.

[15] F. Zampella, M. Khider, P. Robertson, and A. Jimnez, "Unscented Kalman filter and magnetic angular rate update (MARU) for an improved pedestrian dead-reckoning," in Proceedings of the 2012 IEEE/ION Position, Location and Navigation Symposium, April 2012, pp. 129139.

[16] J. Ruppelt, N. Kronenwett, G. Scholz, and G. F. Trommer, "Highprecision and robust indoor localization based on foot-mounted inertial sensors," in 2016 IEEE/ION Position, Location and Navigation Symposium (PLANS), April 2016, pp. 67-75.

[17] O. J. Woodman, "An introduction to inertial navigation," University of Cambridge. Computer Laboratory, Tech. Rep., August 2007, uCAM-CLTR-696 ISSN 1476-2986. 